Original Research Paper

\title{
Performance Evaluation of Channel Propagation Models and Developed Model for Mobile Communication
}

\author{
${ }^{1,2}$ Yahia Zakaria \\ ${ }^{I}$ Department of Electrical Engineering, Faculty of Electrical Engineering and Computer Science, \\ VSB-Technical University of Ostrava, 17.Listopadu 15, 70833 Ostrava, Czech Republic \\ ${ }^{2}$ Engineering Research Division, National Research Centre (NRC), Dokki, P.O. 12622, Cairo, Egypt
}

\author{
Article history \\ Received: 06-12-2016 \\ Revised: 17-02-2017 \\ Accepted: 18-02-2017 \\ Corresponding Author: \\ Yahia Zakaria \\ Department of Electrical \\ Engineering, Faculty of \\ Electrical Engineering and \\ Computer Science, VSB- \\ Technical University of \\ Ostrava, 17, listopadu 15, \\ 70833 Ostrava, Czech Republic \\ Emails: yahia.salem.st@vsb.cz \\ yahia.zk82@yahoo.com
}

\begin{abstract}
Propagation models represent a solidifying of mathematical equations and algorithms that are used for radio signal propagation prediction in specific regions. In this research different propagation models are analyzed and compared. These propagation models have been proposed at the operating frequency of $3.8 \mathrm{GHz}$ for different transmitter antenna heights in all types of terrain. These propagation models depend on location, frequency range and clutter type such as urban, suburban and countryside. We have to bear in mind that the results of the path loss estimation of Free Space model are identical and equal to $(119 \mathrm{~dB})$ for 18 $\mathrm{m}$ and $34 \mathrm{~m}$ transmitter antenna heights at $3.8 \mathrm{GHz}$ in urban environment. It is obvious that Egli model shows the highest path loss values in rural environment as compared with the other models. By the end of this paper, a developed empirical radio propagation model is proposed to be appropriate in urban and rural environments.
\end{abstract}

Keywords: Channel Models, Wireless Communication, Urban, Suburban, Rural

- Large scale fading

- Small scale fading

Existence of the poor sign strength and way deprivation due to the step-down of ability compactness of an electromagnetic wave when it passes through obstruction and multi way propagation environment has been a main defiance over many years in the use of wireless communication systems and this phenomenon is extremely seen in city with many obstacles and high population density (Emagbetere and Edeko, 2009).

The concept that electromagnetic signal has the capacity of propagating over considerable distances with the velocity of igniter was first proposed by (Maxwell, 1865). By added the translation current term to the solidification of par that governing the electromagnetic equations which called now Maxwell's equations, he deduced that among their possible solutions linear wave apparent motion could be included. Hence, the electromagnetic waves should be capable of being propagated over fundamental distances. In 1886, Heinrich Rudolf Hertz observed the transmission of the electromagnetic waves (Schwab and Fischer, 1998).

Thus, multipath fading can be classified into two main types (Jakes, 1974):
Moreover, propagation path loss theoretical account is mathematical shaft that is used by technologist and scientist to plan and enhance radio set communicating systems. A major undertaking in the planning phase of the wireless communication net is to predict the loss of signal strength in a specific location (Isabona and Konyeha, 2013).

The research is arranged as follows: In section 2, the selected models are described whereas in section 3, simulation models are stated. In section 4, the analysis of the results is compared. Then in section 5, the developed empirical model is presented and the conclusions follow in the last section.

The previous work (Zakaria, 2014) compared and analyzed various propagation models (COST 231 Hata Model, Stanford University Interim (SUI) Model and Ericsson Model). These propagation models have been proposed for operating frequency at $2.5 \mathrm{GHz}$ for different receiver antenna heights in all types of the environments (urban, suburban and rural). It was noticed from the results of the path loss estimation for 4 and $8 \mathrm{~m}$ receiver antenna heights in suburban area that SUI model showed the lowest path loss result $(119 \mathrm{~dB}$ in $4 \mathrm{~m}$ 
receiver antenna height) as compared with the other models in suburban environment. On the contrary, Ericsson model showed the highest path loss result (183 $\mathrm{dB}$ in $4 \mathrm{~m}$ receiver antenna height) as compared with the other models in rural environment. Also, COST 231 Hata model showed the highest path loss result (159 dB in $4 \mathrm{~m}$ receiver antenna height) as compared with the other models in urban environment. Moreover, it was mentioned that SUI model showed the lowest path loss result (119 $\mathrm{dB}$ in $4 \mathrm{~m}$ receiver antenna height) as compared with the other models in all types of the environments. It was obvious from the results of the path loss estimation for 4 and $8 \mathrm{~m}$ receiver antenna heights in urban area that SUI and Ericsson models showed the lowest path loss result $(140 \mathrm{~dB}$ in $8 \mathrm{~m}$ receiver antenna height) as compared with the other models in urban environment. On the other hand, COST 231 Hata model showed the highest path loss result (159 dB in $4 \mathrm{~m}$ receiver antenna height) in urban environment.

\section{Description of Selected Models}

\section{Egli Model}

This model can be used to predict the total path loss for a head to point data link. It is calculated by using the following equation (Sumit, 2012):

$$
L_{50}=G_{b} G_{m}\left(h_{b} h_{m} / I^{2}\right)^{2} \beta
$$

Where:

$G_{m}=$ The gain of mobile antenna

$G_{b}=$ The gain of base station antenna

$h_{b}=$ The height of base station antenna

$h_{m}=$ The height of mobile antenna

$l=$ The propagation distance and $\beta=(40 / f)^{2}$

$f \quad=$ The frequency in $\mathrm{MHz}$

\section{Two Ray Ground Reflection (TRGR) Model}

This model considers both the direct itinerary and a dry land reflection path. The received king at distance $d$ is predicted by the following equation (Rani et al., 2014):

$$
P_{r}(d)=\frac{P_{t} G_{t} G_{r} h_{t}{ }^{2} h_{r}{ }^{2}}{d^{4} L}
$$

Where:

$h_{t}$ and $h_{r}=$ The heights of the transmitted and received antennas respectively

$P_{t} \quad=$ The transmitted signal power and $L=1$

$G_{t}$ and $G_{r}=$ The feeler gains of the transmitter and the recipient respectively

\section{Free Space Model}

This model defines how much strength of the signal is lost during the propagation from transmitter to receiver. It is calculated by using the following equation (Alam et al., 2014):

$$
L_{f s}=32.45+20 \log _{10}(d)+20 \log _{10}(f)
$$

Where:

$f=$ The frequency in $\mathrm{MHz}$

$d=$ The distance between transmitter and receiver in meters

\section{Cost 231 Hata Model}

This model is used for application where the root word station transmitting aerial is above certain roof tops and is used widely in radio planning in mobile telephone. The median path loss is given by the following equating (Zakaria, 2014):

$$
\begin{aligned}
& L_{50}(d B)=46.3+33.9 \log \left(f_{c}\right)-13.82 \log \left(h_{t}\right) \\
& -a\left(h_{r}\right)+\left[44.9-6.55 \log \left(h_{t}\right)\right] \log (I)+C
\end{aligned}
$$

\section{Where:}

$f_{c} \quad=$ The frequency in $\mathrm{MHz}$

$h_{t} \quad=$ The base station height in meters

$h_{r}=$ The mobile station height in meters

$a\left(h_{r}\right)=$ The mobile antenna height correction factor

$l \quad=$ The link distance in $\mathrm{km}$

$C=0 \mathrm{~dB}$ for medium cities or suburban centers with medium tree density and equal $3 \mathrm{~dB}$ for metropolitan centers

\section{Young Model}

This model represents the behaviour of cellular communication systems in large metropolis. It is calculated by using the following equation (Eric et al., 2009):

$$
L=G_{B} G_{M}\left[\frac{h_{B} h_{M}}{d^{2}}\right]^{2} \beta
$$

Where:

$L \quad=$ The path loss in $\mathrm{dB}$

$G_{B}=$ The gain of base transmitter in $\mathrm{dB}$

$G_{M}=$ The gain of mobile transmitter in $\mathrm{dB}$

$h_{B}=$ The height of base station antenna in $\mathrm{m}$

$h_{M}=$ The height of base station antenna in $\mathrm{m}$

$d=$ The link distance in $\mathrm{km}$ and $\beta$ is the clutter factor

\section{Simulation Models and Scenarios}

In this section we give more information about simulation models and scenarios by using MATLAB R2015b software. 
Table 1. Parameters of numerical simulations

\begin{tabular}{llll}
\hline Parameters & Urban & Suburban & Rural \\
\hline $\begin{array}{l}\text { Distance between } \\
\text { transmitter and receiver }\end{array}$ & $6 \mathrm{~km}$ & \\
$\begin{array}{l}\text { Mobile transmitter power } \\
\text { Average building height }\end{array}$ & $16 \mathrm{~m}$ & $32 \mathrm{dBm}$ & \\
Transmitter antenna height & & $34 \mathrm{~m}$ & $7 \mathrm{~m}$ \\
Base station transmitter power & $41 \mathrm{mBm}$ & \\
Operating frequency & $3.8 \mathrm{GHz}$ & \\
Receiver antenna height & $12 \mathrm{~m}$ & \\
\hline
\end{tabular}

\section{Simulation Models}

In this research, Egli model, Two Ray Ground Reflection model, Free Space model, Cost 231 Hata model and Young model are analyzed in urban, suburban and rural environments by applying two different transmitter antenna heights. During the simulations the path loss of channel propagation models is estimated by applying two different transmitter antenna height of 12 $\mathrm{m}$. We decided to use the mobile transmitter power equal to the value of $32 \mathrm{dBm}$ by setting up the simulation parameters. By using the script property of MATLAB R2015b software through writing the codes of each simulation, we set the operating frequency for all simulation scenarios to be fixed at $3.8 \mathrm{GHz}$.

\section{Simulation Scenario}

By using run and time property of MATLAB R2015b software through the simulation process of each channel model it was observed that the average time processing for each run is $55 \mathrm{sec}$. In the simulation, we supposed that the distance between transmitter and receiver was applied at this researchat $6 \mathrm{Km}$ and base station transmitter power equal to $41 \mathrm{dBm}$. Moreover, Table 1 shows values of the parameters which were applied at this research. In Simulation Scenario Section, 'run and time property' means the number of each calculation of the path loss. Also, in Simulation Scenario Section, 'each run' means the calculation by using the mentioned parameters as indicated in Table 1 , as operating frequency and distance between transmitter and receiver are fixed at the values of $3.8 \mathrm{GHz}$ and $6 \mathrm{~km}$, respectively.

Simulations were performed with six trials. In each trial, the value of transmitter antenna height was changed according to the type of environment. During each trial, path loss was calculated by using all the former mentioned parameters in urban, suburban and rural environments.

\section{Results Analysis and Discussion}

In this study, we calculated and analyzed various propagation models (Egli, TRGR, Free Space, COST 231 Hata, Young) and a developed empirical radio propagation model is proposed to be suitable in urban and rural environments. These propagation models have been proposed at the operating frequency of $3.8 \mathrm{GHz}$ for different transmitter antenna heights in all types of terrain. The results of the path loss estimation of Free Space model are identical and equal to $(119 \mathrm{~dB})$ for 18 and $34 \mathrm{~m}$ transmitter antenna heights at $3.8 \mathrm{GHz}$ in urban environment. It is obvious that Egli model shows the highest path loss values in rural environment as compared with the other models. Two ray ground reflection model showed the highest values $(174.4 \mathrm{~dB})$ and $(169.3 \mathrm{~dB})$ in 6 and $10 \mathrm{~m}$ receiver antenna heights, respectively in urban environment at $3.5 \mathrm{GHz}$. However, attention should be paid to the results of the path loss estimation of Free Space model which are identical and equal to $(109.4 \mathrm{~dB})$ and $(106.4 \mathrm{~dB})$ at 3.5 and $2.5 \mathrm{GHz}$, respectively in urban environment.

In the computation, operating frequency and distance between transmitter and receiver are fixed at the values of $3.8 \mathrm{GHz}$ and $6 \mathrm{~km}$, respectively. Two different transmitter antenna heights (34 and $18 \mathrm{~m}$ ) are considered in this case. The value of receiver antenna height are fixed at $12 \mathrm{~m}$ for the above mentioned calculation. All the other parameters which are used in this research are described in Table 1.

The numerical results of the selected models in urban area for different transmitter antenna heights are shown in Fig. 1 and 2.

It was obvious from (Alam et al., 2014) that the results of Free Space model stated the lowest value $(106.4 \mathrm{~dB})$ at $2.5 \mathrm{GHz}$ in the same environment. Two ray ground reflection model showed the highest values $(174.4 \mathrm{~dB})$ and $(169.3 \mathrm{~dB})$ in 6 and $10 \mathrm{~m}$ receiver antenna heights, respectively in urban environment at 3.5 $\mathrm{GHz}$. However, attention should be paid to the results of the path loss estimation of Free Space model which are identical and equal to $(109.4 \mathrm{~dB})$ and $(106.4 \mathrm{~dB})$ at 3.5 and $2.5 \mathrm{GHz}$, respectively in urban environment.

The numerical results of the models in suburban area for different transmitter antenna heights are shown in Fig. 3 and 4.

By comparing the results in (Alam et al., 2014) with these results, it was noticed that the result of Egli model had the lowest value $(160.2 \mathrm{~dB})$ at $3.5 \mathrm{GHz}$ in the same environment. Egli model showed the highest values (158.2 $\mathrm{dB})$ and $(156.8 \mathrm{~dB})$ in $6 \mathrm{~m}$ and $10 \mathrm{~m}$ receiver antenna heights, respectively in suburban environment at $3.5 \mathrm{GHz}$. However, attending should be paid to the outcome of the path loss appraisal of Free Space model which are identical and equal to $(109.4 \mathrm{~dB})$ and $(106.4 \mathrm{~dB})$ at 3.5 and $2.5 \mathrm{GHz}$, respectively in all types of environments.

The numerical results of the models in rural area for different transmitter antenna heights are shown in Fig. 5 and 6.

By comparing the results in (Vyas, 2014) with these results, it has found that the results of Free Space model and Cost 231 Hata model in the mentioned paper stated the lowest value $(113.5,149.3,164.5$ and $158 \mathrm{~dB})$, respectively in 3,6 and $10 \mathrm{~m}$ receiver antenna heights in urban environment at $4.5 \mathrm{GHz}$. 


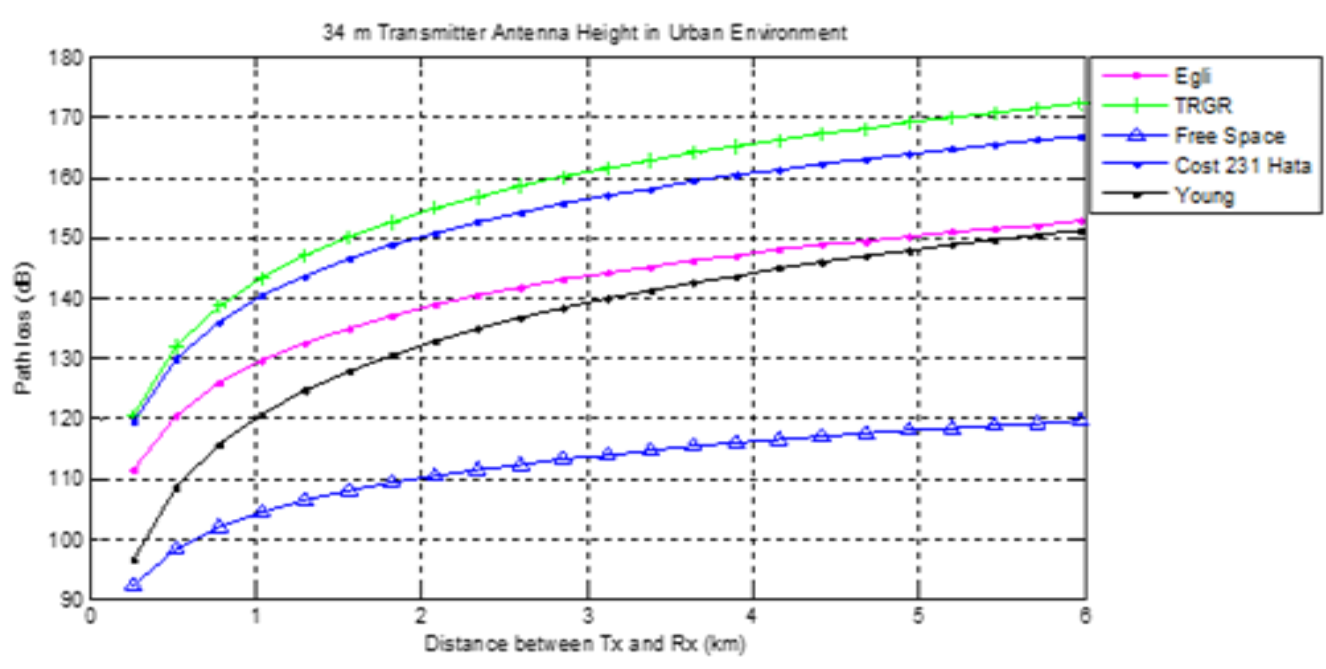

Fig. 1. Path loss for $34 \mathrm{~m}$ transmitter antenna height in urban environment

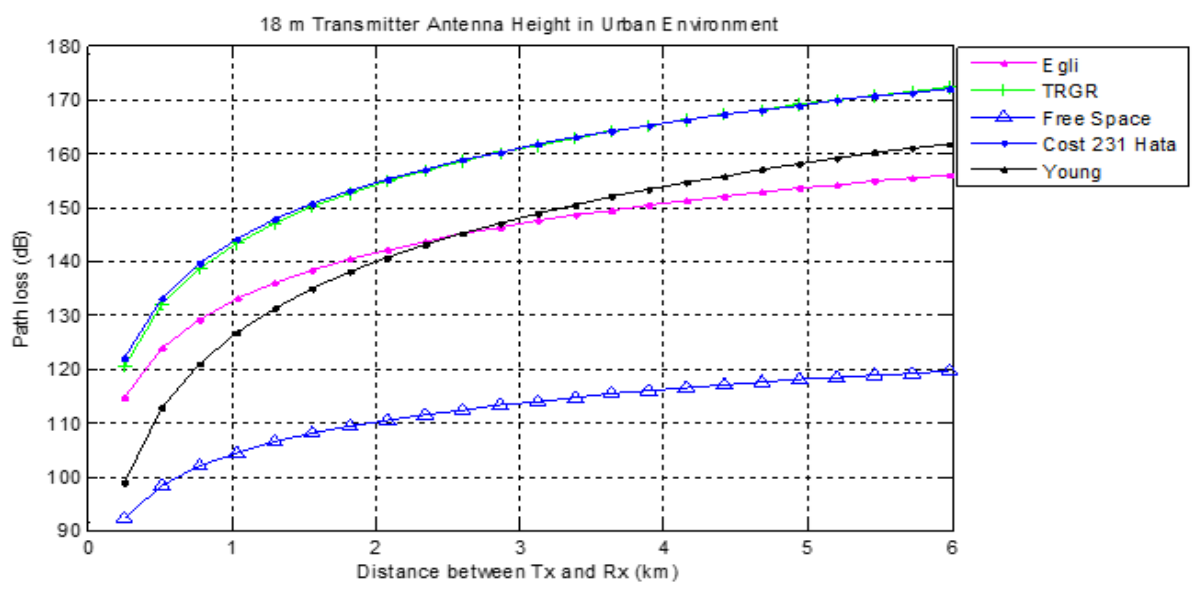

Fig. 2. Path loss for $18 \mathrm{~m}$ transmitter antenna height in urban environment

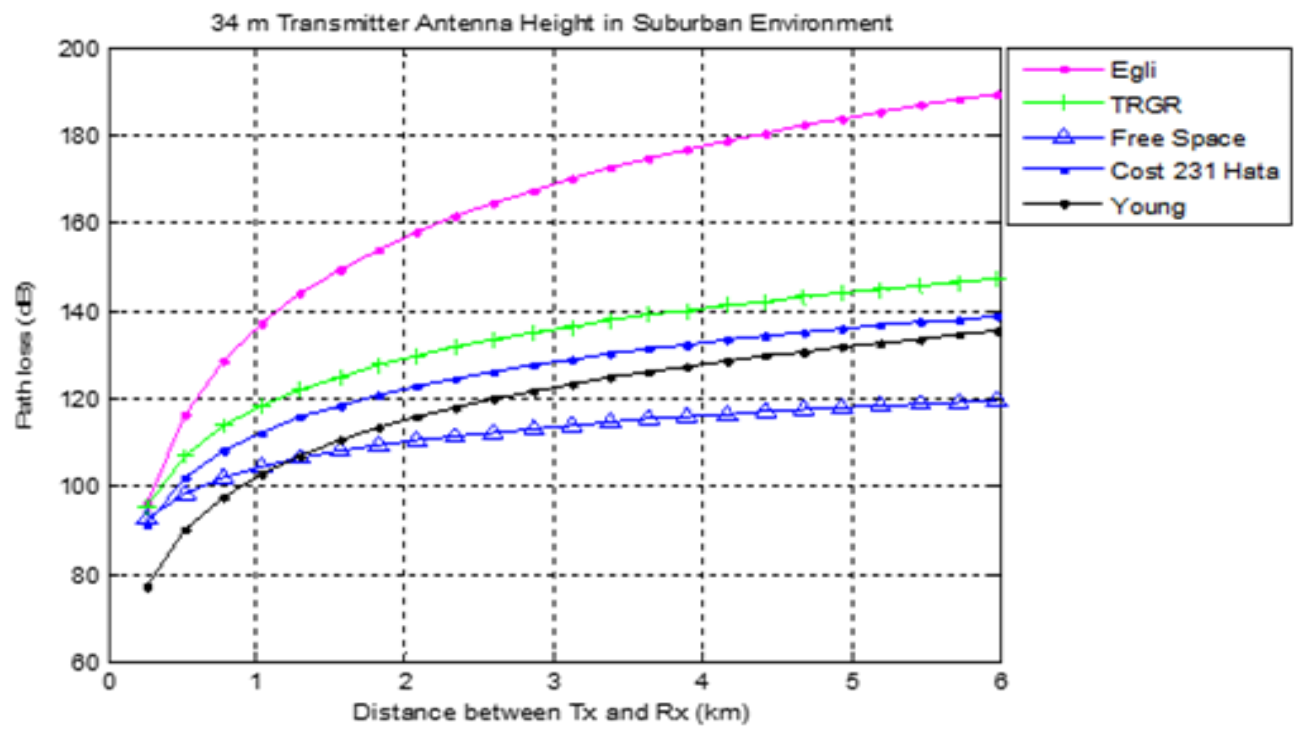

Fig. 3. Path loss for $34 \mathrm{~m}$ transmitter antenna height in suburban environment 


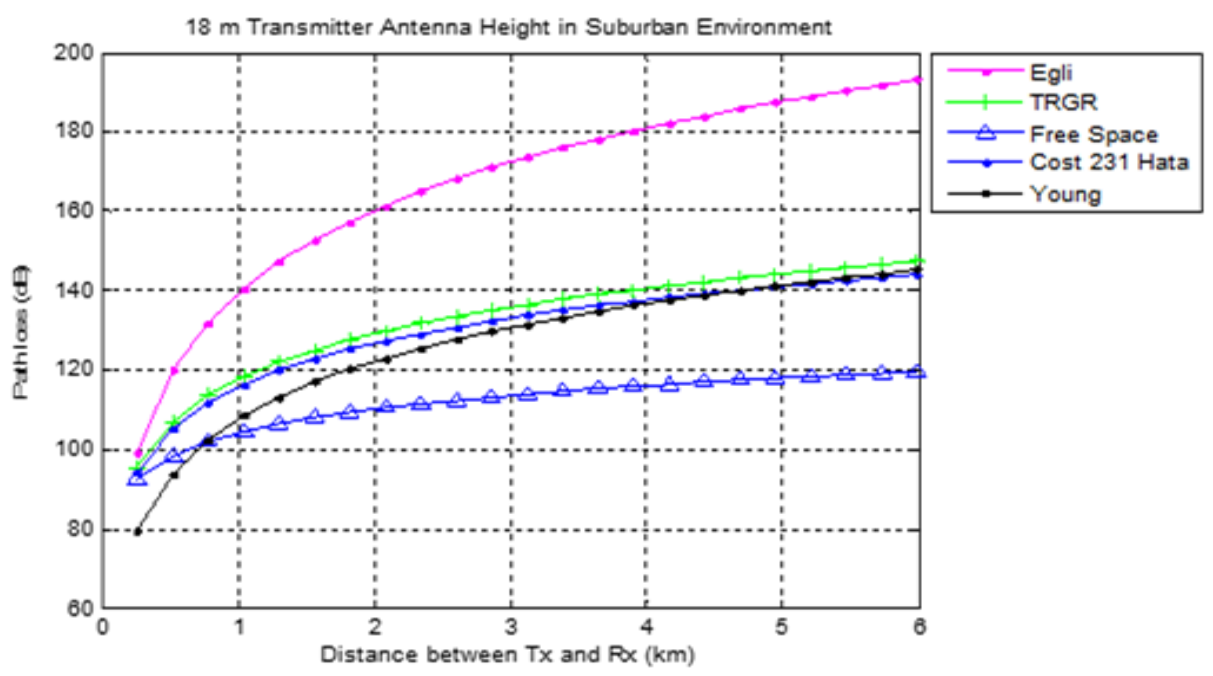

Fig. 4. Path loss for $18 \mathrm{~m}$ transmitter antenna height in suburban environment

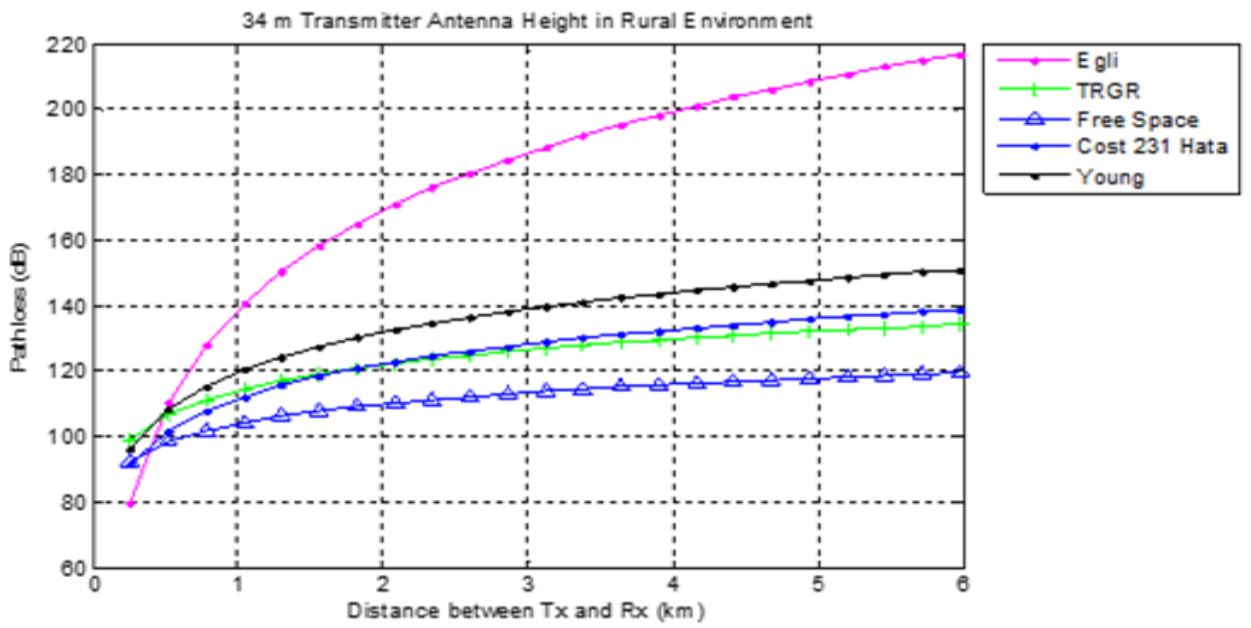

Fig. 5. Path loss for $34 \mathrm{~m}$ transmitter antenna height in rural environment

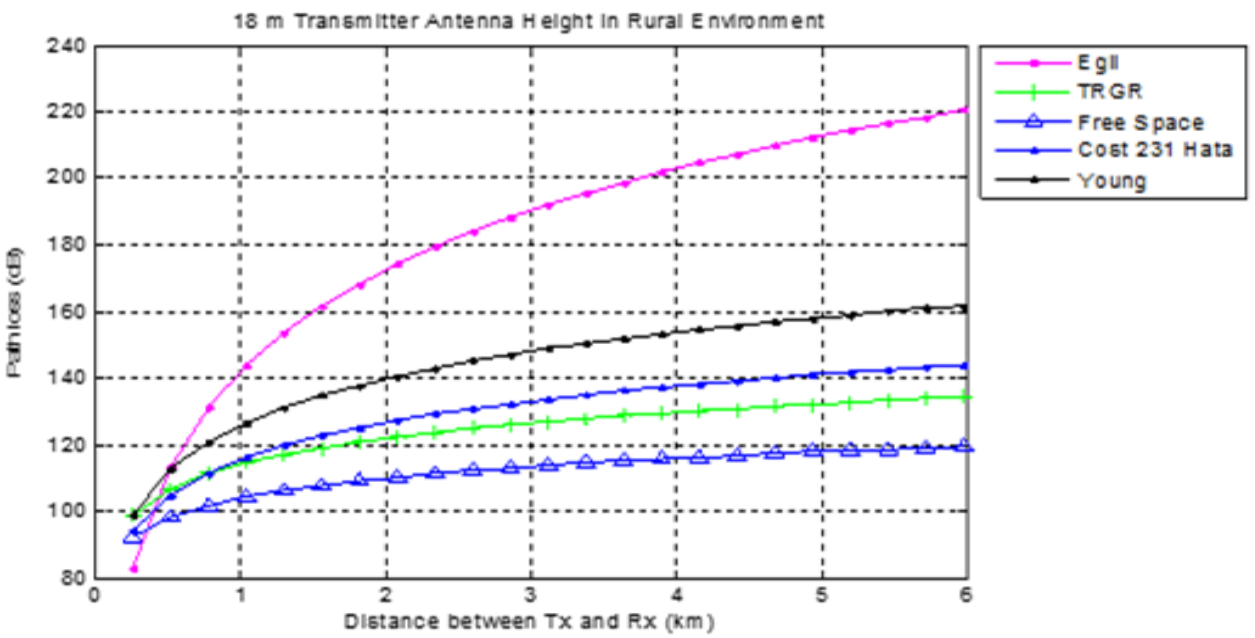

Fig. 6. Path loss for $18 \mathrm{~m}$ transmitter antenna height in rural environment 


\section{Urban Environments}

घ $34 \mathrm{~m}$ Tx Height \& $3.8 \mathrm{GHz} \quad \square 18 \mathrm{~m}$ Tx Height \& $3.8 \mathrm{GHz}$

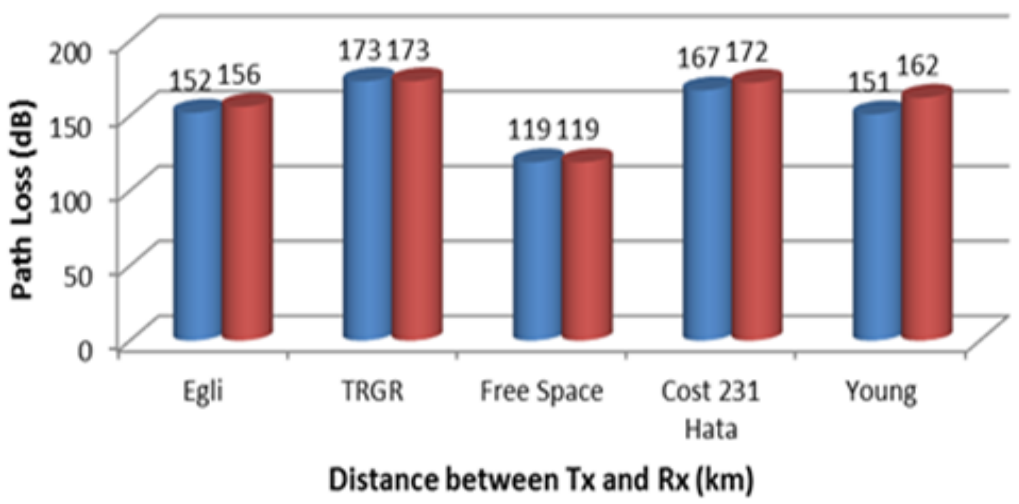

Fig. 7. Analysis of the results in urban environment

\section{Suburban Environments}

= $34 \mathrm{~m}$ Tx Height \& $3.8 \mathrm{GHz}=18 \mathrm{~m} \mathrm{Tx}$ Height \& $3.8 \mathrm{GHZ}$

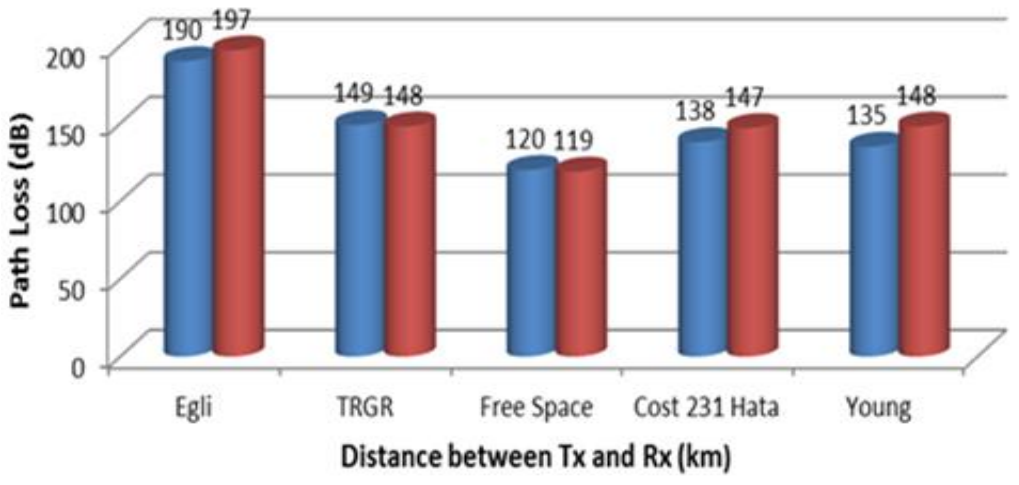

Fig. 8. Analysis of the results in suburban environment

\section{Rural Environments}

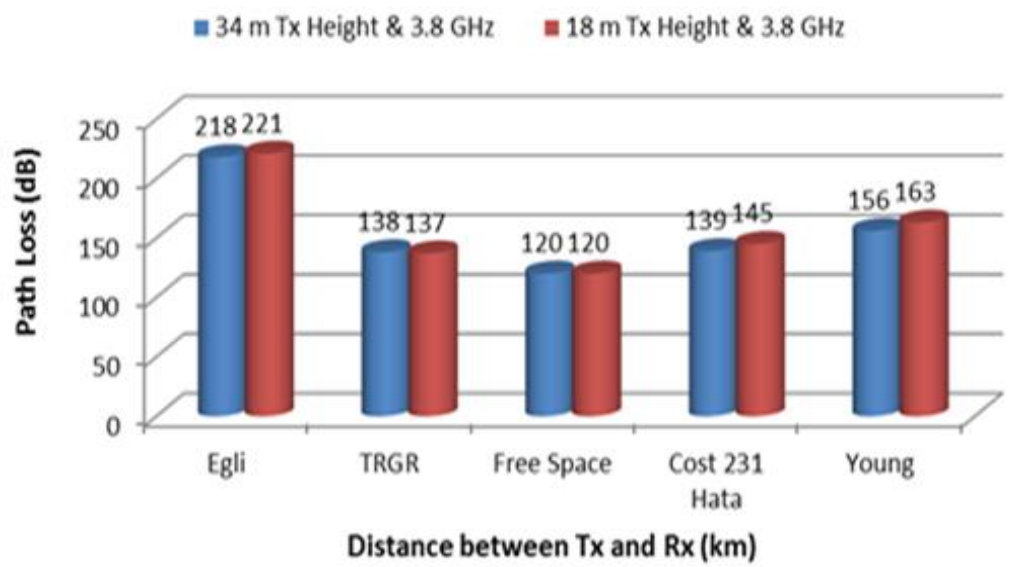

Fig. 9. Analysis of the results in rural environment 
However, attention should be paid in this research to the results of the path loss estimation of Free Space model which are identical and equal to $(119 \mathrm{~dB})$ for 18 and $34 \mathrm{~m}$ transmitter antenna heights at $3.8 \mathrm{GHz}$ in urban environment.

By comparing these results with (Vyas, 2014), we can observe that the Free Space model showed the lowest result in suburban area at $4.5 \mathrm{GHz}$. Moreover, it was noticed from the results of the path loss estimation for 3,6 and $10 \mathrm{~m}$ receiver antenna heights in suburban environment that ECC-33 model showed the highest path loss result (176.3 $\mathrm{dB}$ in $3 \mathrm{~m}$ receiver antenna height) as compared with the other models in suburban environment.

By comparing the results in (Vyas, 2014) with these results, it has found that the results of Cost $231 \mathrm{~W}-\mathrm{I}$ model in the mentioned paper stated the lowest value $126 \mathrm{~dB}$ in 3, 6 and $10 \mathrm{~m}$ receiver antenna heights in rural environment at $4.5 \mathrm{GHz}$.

Furthermore, it can be mentioned that the results of the path loss estimation in this research of Young model $(163 \mathrm{~dB})$ for $18 \mathrm{~m}$ transmitter antenna height in suburban environment are identical and equal to the results of the path loss estimation of Young model for the same transmitter antenna height in rural environment. However, attending should be paid to the outcome of the path loss appraisal of Free Space model which are identical and equal to $(120 \mathrm{~dB})$ for $18 \mathrm{~m}$ and $34 \mathrm{~m}$ transmitter antenna heights in rural environment.

By observing the above graphs of this research, It was obvious from the results of the path loss estimation for 18 and $34 \mathrm{~m}$ receiver antenna heights in rural environment that Free Space model showed the lowest path loss results $(120 \mathrm{~dB}$ in 18 and $34 \mathrm{~m}$ transmitter antenna heights) as compared with the other models in rural environment. Moreover, it was observed that Egli model showed the highest path loss result $(221 \mathrm{~dB}$ in 18 $\mathrm{m}$ transmitter antenna height) as compared with the other models in rural environment.

The results analysis of the path loss values for various propagation models in urban, suburban and rural environments are shown in Fig. 7-9, respectively.

By comparing the results in (Mahesh and Rao, 2014) with these results, it has found that the results of Cost $231 \mathrm{~W}-\mathrm{I}$ model in the mentioned paper stated the highest value $(176.22 \mathrm{~dB})$ in urban environment at $4.2 \mathrm{GHz}$. On the contrary, Free Space model showed the lowest value 119.37 dB in urban environment at $3.7 \mathrm{GHz}$.

However, attending should be paid to the outcome of the path loss appraisal of Free Space model which are identical and equal to (119.37 and $120.47 \mathrm{~dB})$ at 3.7 and $4.2 \mathrm{GHz}$, respectively in urban, suburban and rural environments.

By comparing these results with (Mahesh and Rao, 2014), we can observe that the Free Space model showed the lowest result in suburban area $(117 \mathrm{~dB})$ at $2.3 \mathrm{GHz}$.

Moreover, it was noticed from the results of the path loss estimation of the mentioned paper that Free Space model showed the lowest result $(119.37 \mathrm{~dB})$ at $3.7 \mathrm{GHz}$ in suburban environment.

So, the $\mathrm{x}$-axis of Fig. 7 (and Fig. 8 and 9 as well) is the distance between the transmitter and the receiver, but it is labeled with propagation model names, because the $\mathrm{x}$-axis of these figures for the required analysis refer to both of the distance between the transmitter and the receiver and the names of the propagation models.

By observing the above analysis of this research, it was obvious from the results of the path loss estimation that Egli model showed the highest path loss results (221 $\mathrm{dB})$ as compared with the other models in rural environment at $3.8 \mathrm{GHz}$.

\section{Developed Empirical Model}

The proposed model is developed to be appropriate for mobile communication as the interaction between the electromagnetic wave and the environs reduces the signal strength which is sent from transmitter to pass receiver that causes the track release as the transfer of information between the transmitting antenna and the receiving antenna is achieved by means of electromagnetic waves. Moreover, existence of the poor people signal posture and path loss due to the decrease of office density of an electromagnetic wafture when it crack through obstacle and multi path generation environment has been a main defiance over many year in the use of wireless communication systems and this phenomenon is extremely seen in metropolis with many obstacles and senior high population density. Also, the path loss may be caused due to many effects like free space loss, diffraction, reflection and absorption. The path loss prediction may differ from one propagation model to another one due to the differences in city structures, local terrain profiles and weather.

The proposed model was designed based on the concept of the path loss as the measure of average radio frequency attenuation occurred by a transmitted signal when it arrives finally to the receiver and usually defined as the following equation (Bertoni, 2000):

$$
P L(d)=P L\left(d_{0}\right)+10 n \log _{10}\left(\frac{d}{d_{0}}\right)
$$

Where:

$d=$ The distance

$d_{0}=$ The reference point at $1 \mathrm{~km}$

$n=$ The path loss exponent

In free space, the power arriving the receiving antenna which is separated from the transmitting antenna by a distance $d$ is given by the following Fri is free space equation: 


$$
P_{r}=\frac{P_{t} G_{r}^{2} G_{t}}{d^{2} L(4 \pi)^{2}}(d)
$$

Where:

$G_{t}$ and $G_{r}=$ The gain of the transmitting and receiving antenna, respectively

$L=$ System loss factor

$\lambda=\quad$ The wavelength in meters

Moreover, existence of the poor signal strength and path loss due to the reduction of power density of an electromagnetic wave when it passes through obstacles and multi path propagation environment has been a main defiance over many years in the use of wireless communication systems and this phenomenon is extremely seen in cities with many obstacles and high population density. Also, the path loss may be caused due to many effects like free space loss, diffraction, reflection and absorption. The path loss prediction may differ from one propagation model to another one due to the differences in city structures, local terrain profiles and weather.

The great growth in mobile communication systems is due to development of highly reliable devices and the development of the cellular concept. The cellular concept was a main character in solving the job of spectral crowding and user capacity. It offered high capacity with a limited spectrum allocation without any prime technological changes. To enclose a good quality of the transmission path of the signal, the signal received in a cell must be strong. Once the signal has crossed the bound of a cell, it becomes incumbrancer. Thus, the channel frequency is usually not reused in neighboring cells. If it is reused, the co-channel interference may damage reception of the signal in the adjacent cells and the quality of the service may strictly degrade.

By taking into account that our obtained results from this research by using the described propagation models (Egli, TRGR, Free Space, COST 231 Hata, Young) for calculating the path loss based on the stated parameters as Mobile transmitter power, Distance between transmitter and receiver, Average building height, Transmitter antenna height, Base station transmitter power, Operating frequency, Receiver antenna height, it is found that the proposed model is more accurately represents the signal propagation for mobile communication in rural and urban environments. Therefore, based on the above comparison and analysis, the following empirical model is developed to be suitable in urban and rural environments:

$$
P L=2.56+20 n \log _{10} d+24 h_{r}+12 h_{t}+C
$$

Where:

$P L=$ The median path loss in $\mathrm{dB}$

$h_{t}=$ The transmitter height in meters $d=$ The distance from the transmitter in meters

$n=$ The path loss exponent

$h_{r}=$ The receiver height in meters

$C=$ The factor of specific environment

$$
C=\left\{\begin{array}{lr}
2 & \text { for urban } \\
5 & \text { for rural }
\end{array}\right.
$$

\section{Conclusion and Future Work}

The main goal of the propagation models is to predict the loss of signaling strength or reportage in a particular location. Moreover, they are mathematical instrument used by engineers and scientists to plan and optimize wireless communicating arrangement. In this research the behavior of channel multiplication models of wireless communicating systems is analysed and a developed empirical model is presented. The most significant factor during the planning phase of radio set communication systems is to predict the loss of signal strength in a specific strength in a specific environment. As a conclusion in the typesetter's case examined here, it can be noticed that Free Space Infinite model showed the lowest path deprivation model showed the lowest path loss results as compared with the other models in all types of terrain. Finally, it is necessary to distributor point out that the interaction between the electromagnetic Wave and the environment reduces the sign strength which is sent from vector to receiver which causes the path loss. In the near future, we would like to compare the measurements from suburban area with both of Egli and COST 231 Hata models. Then, the obtained results should be analyzed in order to find out which model is better in urban environment and which one is better in suburban environment. Then, we may try to find appropriate parameters for COST 231 Hata model.

\section{Acknowledgement}

The support of authors by VSB-Technical University of Ostrava, Czech Republic was acknowledged.

\section{Funding Information}

This research is supported by the project SP 2016/143 "Research of antenna systems; effectiveness and diagnostics of electric drives with harmonic power; reliability of the supply of electric traction; issue data anomalies". The authors would like to express their thanks to the members of Department of Electrical Engineering at VSB-Technical University of Ostrava for their support.

\section{Ethics}

The authors have read and approved the manuscript and give the assurance that no part of this original 
research article is being considered for publication in whole or in part elsewhere.

\section{References}

Alam, M.D., S. Chowdhury and S. Alam, 2014. Performance evaluation of different frequency bands of WiMAX and their selection procedure. Int. J. Adv. Sci. Technol., 62: 1-18. DOI: 10.14257/ijast.2014.62.01

Bertoni, H., 2000. Radio Propagation for Modern Wireless Systems. 1st Edn., Prentice Hall PTR, Upper Saddle River, ISBN-10: 0130263737, pp: 258.

Emagbetere, J.O. and F.O. Edeko, 2009. Measurement validation of hata-like models for radio propagation path loss in rural environment at $1.8 \mathrm{GHz}$. J. Mob. Commun., 3: 17-21.

Eric, A., C. Phillips, D. Sicker and D. Grunwald, 2009. Modeling environmental effects on directionality in wireless networks. Proceedings of the 5th International Workshop on Wireless Network Measurements, (WNM' 09).

Isabona, J. and C.C. Konyeha, 2013. Urban area path loss propagation prediction and optimisation using hata model at $800 \mathrm{MHz}$. J. Applied Phys., 3: 8-18.
Jakes, W.C., 1974. Microwave Mobile Communications. 1st Edn., IEEE Press, Piscataway, ISBN-10: 0780310691, pp: 642.

Mahesh, B.C. and B.P. Rao, 2014. Design and modeling of propagation models for WiMAX communication system at $3.7 \mathrm{GHz}$ and $4.2 \mathrm{GHz}$. Int. J. Electron. Commun., 2: 1-7.

Maxwell, J.C., 1865. A dynamical theory of the electromagnetic field. Philos. Trans. Roy. Soc. Lond., 155: 459-512. DOI: 10.1098/rstl.1865.0008

Rani, P., V. Chauhan, S. Kumar and D. Sharma, 2014. A review on wireless propagation models. Int. J. Eng. Innovat. Technol., 3: 256-261.

Schwab, A.J. and P. Fischer, 1998. Maxwell, hertz and german radio-wave history. Proc. IEEE, 86: 13121318. DOI: $10.1109 / 5.681365$

Sumit, J., 2012. Outdoor propagation models a literature review. Int. J. Comput. Sci. Eng., 4: 281-291.

Vyas, M., 2014. Optimization of empirical path loss models of WiMax at $4.5 \mathrm{GHz}$ frequency band. IOSR J. Electron. Commun. Eng., 9: 1-8.

Zakaria, Y., 2014. Performance analysis of propagation models for cellular mobile communication systems at 2.5 GHz. Int. J. Scientific Eng. Res., 5: 426-432. 